\title{
FIBROMA OSSIFICANTE CENTRAL EM CRIANÇA: RELATO DE CASO
}

\author{
CENTRAL OSSIFYING FIBROMA IN CHILD: CASE REPORT
}

\author{
Lucas Teixeira BRITO'; Germano ANGARANI'; Angela Beatriz Cavalcante de Amorim IZAC2; Rubens Jorge SILVEIRA ${ }^{3}$ \\ 1 - Cirurgião-dentista. Residente no Departamento de Cirurgia e Traumatologia Buco-Maxilo-Facial, Hospital Estadual Governador Otávio Lage de \\ Siqueira (HUGOL), Goiânia, Goiás, Brasil. \\ 2 - Cirurgiã Bucomaxilofacial. Departamento de Cirurgia e Traumatologia Buco-Maxilo-Facial, Hospital Estadual Governador Otávio Lage de \\ Siqueira (HUGOL), Goiânia, Goiás, Brasil. \\ 3 - Mestre em Odontologia pela Universidade Federal de Uberlândia (UFU). Departamento de Cirurgia e Traumatologia Buco-Maxilo-Facial. \\ Hospital Estadual Governador Otávio Lage de Siqueira (HUGOL), Goiânia, Goiás, Brasil. Faculdade São Leopoldo Mandic, Institutos de \\ Pesquisas São Leopoldo Mandic, Área de Imaginologia Odontológica, Campinas, São Paulo, Brasil. Departamento de Cirurgia Oral e Maxilofacial, \\ Universidade Paulista, Goiânia, Goiás, Brasil.
}

\section{RESUMO}

Objetivo: O fibroma ossificante central, de acordo com a última classificação da OMS, é um tumor de origem odontogênica, por apresentar origem nas células do ligamento periodontal. A maior parte das lesões são encontradas nas regiões próximas aos dentes e com mais frequência em mandíbula. Acometem mais o sexo feminino e a idade mais incidente é por volta da terceira década de vida. Achados imaginológicos e/ou assimetrias faciais são as principais suspeitas. O diagnóstico deve incluir informações da anamnese, bem como exames de imagem e histopatológico que confirma a natureza da lesão. $O$ tratamento pode variar desde uma curetagem da lesão até ressecção em bloco de acordo com o tamanho da lesão e sua localização. Os autores descrevem o caso clínico de uma paciente de 13 anos, sexo feminino, com lesão unilateral em mandíbula. Material e método:
O tratamento proposto, após confirmação da lesão através da biópsia incisional, foi a exérese da lesão com preservação do nervo mentual, realizado sob anestesia local. Com 6 meses de acompanhamento, a lesão se mostrou persistente, porém com alterações mediante novo histopatológico. Resultados: Diante da ausência de expansão da lesão e a idade da paciente, bem como seu desenvolvimento ósseo, optou-se pela proservação do caso, com acompanhamentos semestrais. Conclusões: As lesões fibro-ósseas devem ser tratadas de forma individualizada e de acordo com a vivência do cirurgião, haja vista que por apresentarem curso benigno, as ressecções amplas devem ser ponderadas, nesse caso se faz necessário discutir as condutas frente a essas lesões.

Palavras-Chave: Fibroma ossificante; Mandíbula; Neoplasias.

\section{INTRODUÇÃO}

O fibroma ossificante central, de acordo com a última classificação da Organização Mundial da Saúde (OMS) ${ }^{1}$, é um tumor de origem odontogênica, por apresentar origem nas células do ligamento periodontal e caracterizado pela substituição óssea normal por tecido do tipo fibroso com variados graus de mineralização que acometem, principalmente, os ossos maxilares ${ }^{2,3}$.

Epidemiologicamente, há uma predileção pelo sexo feminino, acometendo principalmente a terceira década de vida. A mandíbula apresenta uma incidência cinco vezes maior quando comparada à maxila. Clinicamente, os pacientes apresentam expansão da cortical óssea no sentido bucolingual, deslocamento dentário e reabsorção radicular ${ }^{4}$. O crescimento, em geral, é de forma lenta e indolor, porém em alguns casos pode causar assimetria facial, dor local, perda dentária, reabsorção radicular e alteração da oclusão ${ }^{5,6}$. Em exames radiográficos, se apresentam classicamente como uma lesão bem definida, radiolúcida com radiopacidades dependendo do seu estágio de evolução ${ }^{1,3,4}$. Em crianças, é uma lesão rara que frequentemente ocorre entre os 5 e 15 anos de idade, correspondendo a $2 \%$ dos tumores orais em crianças $^{5,6}$.

Devido a sua natureza localmente agressiva, a lesão apresenta alto risco de recorrência, necessita ser compretamente enucleada do osso circundante ${ }^{5,6}$. Alguns autores trazem a curetagem como o método de tratamento mais conservador ${ }^{5}$. Com este trabalho os autores tiveram por objetivo descrever um caso clínico de fibroma ossificante central juvenil localizado em região de corpo mandibular.

\section{RELATO DE CASO}

Paciente do sexo feminino, 13 anos, sem queixas sintomatológicas ou assimetrias faciais foi encaminhada pelo ortodontista para avaliação da lesão constatada durante os exames radiográficos para tratamento ortodôntico.

Ao exame intraoral, foi constatado ausência do dente 44 e um leve aumento no fundo de sulco de vestíbulo mandíbular direito (Figura 1). No exame tomográfico, notou-se uma imagem hiperdensa unilocular, bem delimitada, com diferentes graus de hipodensidade, giroversão do dente 43 , impacção do dente 44 e 
mesialização do dente 45. Em cortes transversais, a lesão compreendia toda a extensão vestíbulo-lingual com cortical óssea intacta e sem abaulamento (Figura 2).

Após a biópsia incisional e confirmação do tumor odontogênico, foi realizada exérese da lesão, exodontia do dente associado e ostectomia periférica, sob anestesia local utilizando lidocaína 2\% com epinefrina 1:100.000, através de incisão trapezoidal que contemplou desde a mesial do dente 42 até a mesial do dente 46 com lâmina de bisturi $\mathrm{n}^{\mathrm{o}} 15$. Após descolamento mucoperiosteal foi confeccionada uma demarcação na cortical vestibular da mandíbula, com base na tomografia, usando broca cirúrgica tronco cônica $\mathrm{n}^{\mathrm{o}} 701$ e união dos pontos para confeccionar uma canaleta servindo de apoio para alavanca (Figura 3).

Após remoção do tecido lesionado e do dente envolvido pela lesão, foi realizada a curetagem e em seguida, a ostectomia periférica da loja óssea com broca esférica carbide no 6 em peça reta cirúrgica W\&H (Figura 4). Irrigação abundante com solução fisiológica estéril e sutura com fio de nylon agulhado 5-0 (Figura 5). A peça cirúrgica foi encaminhada para a análise histopatológica e o laudo confirmou a hipótese diagnóstica de Fibroma Ossificante Central (Figura 6).

Após 6 meses, um novo exame tomográfico evidenciou recidiva da lesão (Figura 7). Novo procedimento de biópsia foi realizado sob anestesia local de lidocaína 2\% com epinefrina 1:100.000 com broca trefina de $6 \mathrm{~mm}$ acoplada ao motor de implante NSK e o material foi encaminhado para exame histopatológico que apresentou redução da quantidade de trabéculas osteóides em comparação com o exame anterior. Levando em consideração o laudo do histopatológico pós-operatório, a não expansão da lesão e a idade da paciente, bem como seu desenvolvimento ósseo, optou-se pela proservação do caso, com acompanhamentos semestrais (Figura 8).

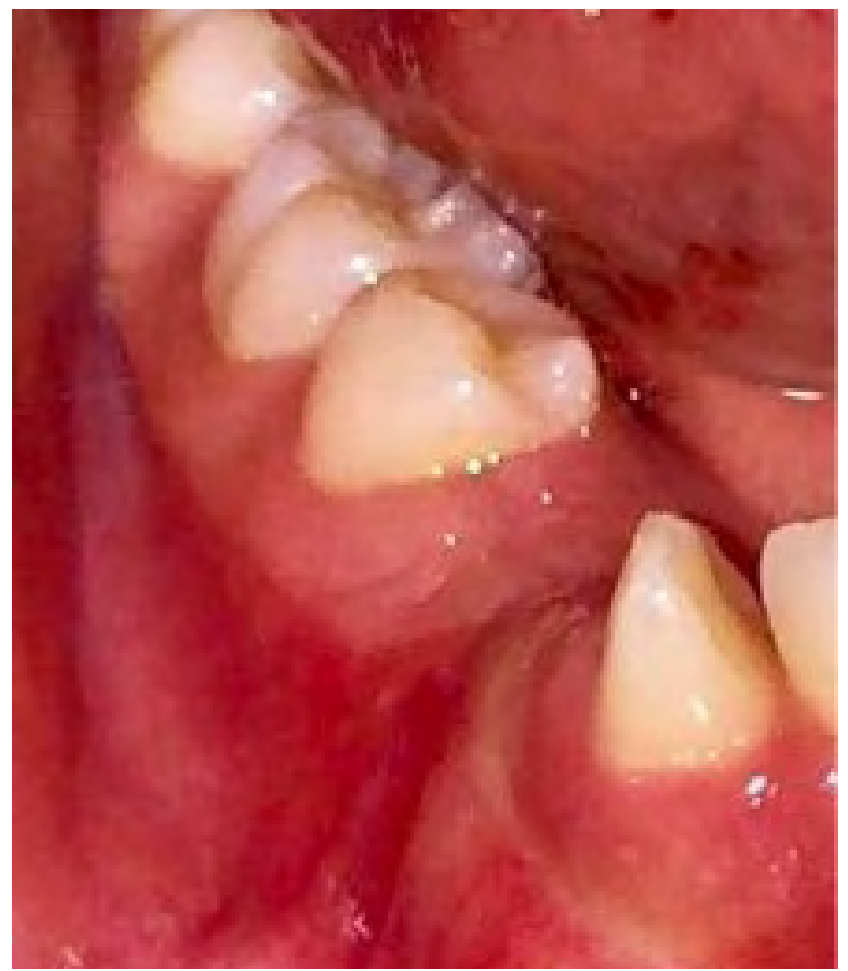

Figura 1 - Aspecto intraoral evidenciando ausência do dente 44 e aumento do fundo de sulco vestibular direito

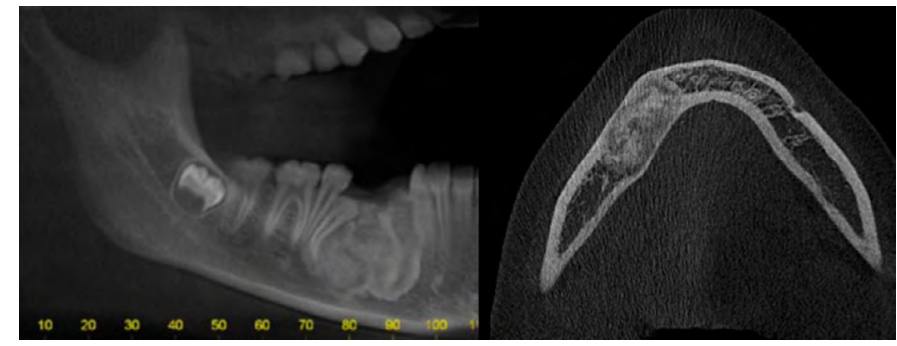

Figura 2 - Imagens tomográficas da lesão

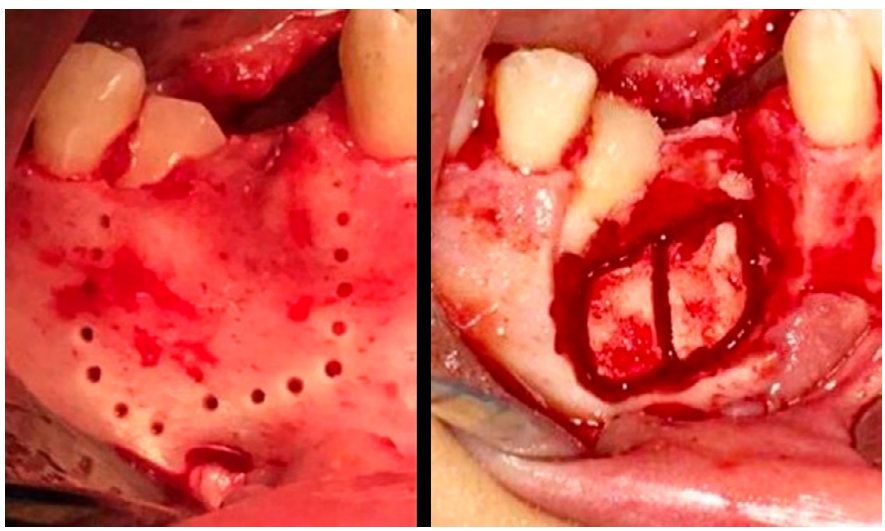

Figura 3 - Demarcação da cortical vestibular e união dos pontos

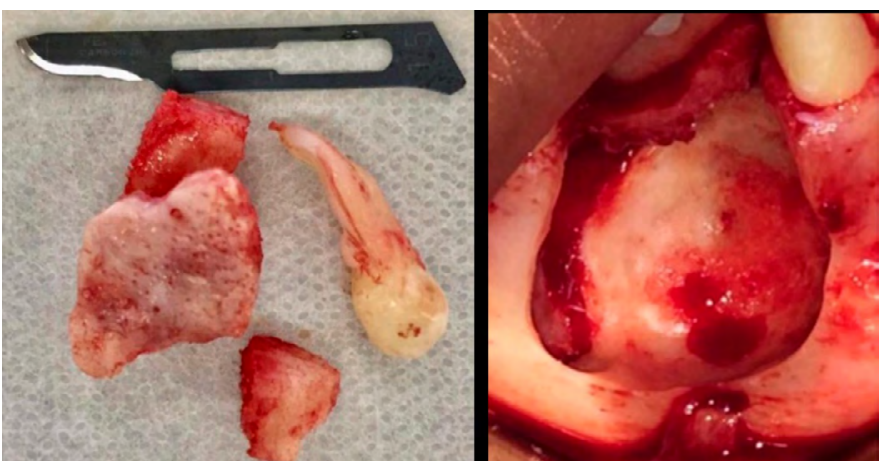

Figura 4 - Aspecto após curetagem da lesão e ostectomia periférica

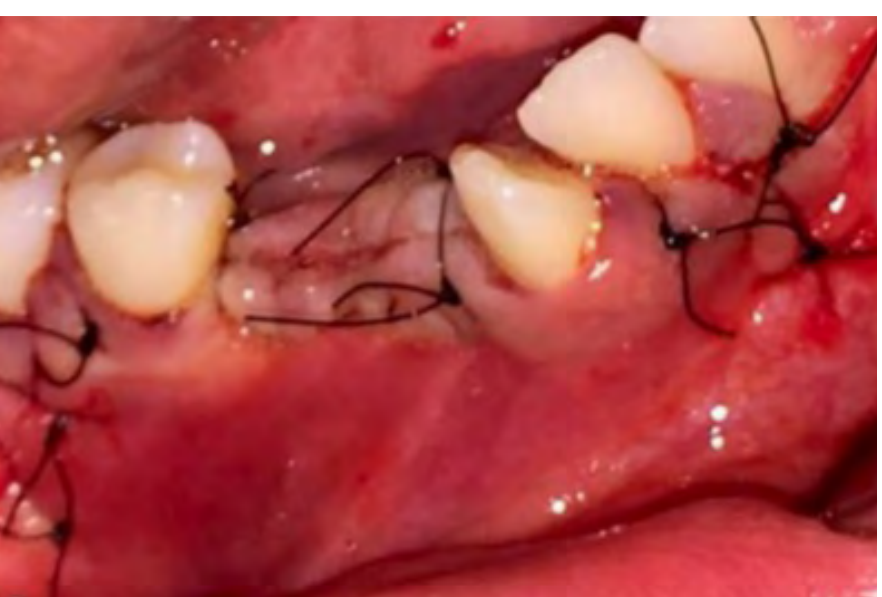

Figura 5 - Aspecto pós-operatório imediato 


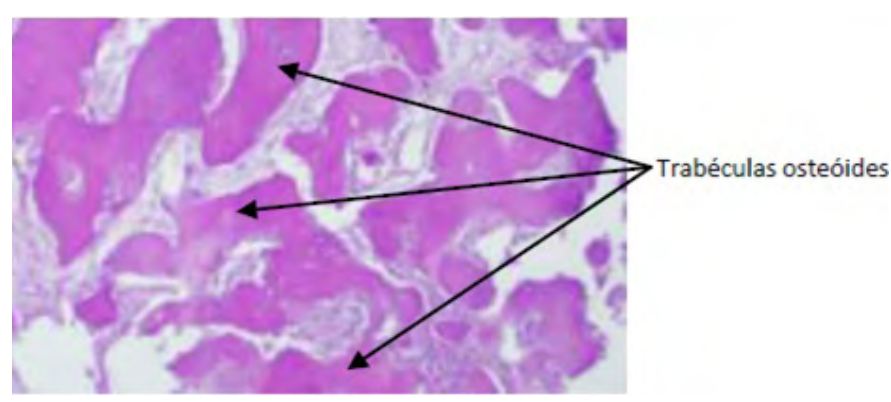

Figura 6 - Exame microscópico revelando diagnóstico de Fibroma Ossificante Central



Figura 7 - Tomografia evidenciando recidiva da lesão

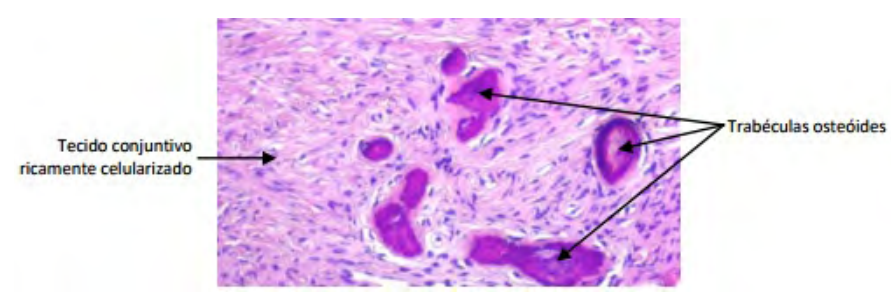

Figura 8 - Laudo histopatológico de controle

\section{DISCUSSÃO}

O Fibroma Ossificante Central (FOC) é um tumor odontogênico que afeta os maxilares, principalmente a mandíbula. O acometimento de ambas as arcadas é raramente descrito na literatura ${ }^{5-7}$.

O sexo feminino, com predileção pela terceira década de vida é o mais acometido, porém, há relatos na literatura de lesões em pacientes mais jovens, como o caso apresentado no presente $\operatorname{artigo~}^{3,8,9}$.

FOC normalmente tem desenvolvimento assintomático, sendo diagnosticados em exames radiográficos de rotina ou por assimetrias faciais. Sintomatologia dolorosa é mais comumente relatada no Fibroma Ossificante Juvenil, que apresenta características mais agressivas quando comparadado ao FOC. Apesar de o caso clínico ter ocorrido em uma jovem de 13 anos, não se apresentou agressivo/expansivo, permitindo uma abordagem mais conservadora ${ }^{9,10}$.

Os aspectos radiográficos se alteram de acordo com o estágio de desenvolvimento da lesão, podendo apresentar-se radiolúcidos, radiopacos e com mais frequência apresentando um padrão misto de radiolucidez com pontos de radiopacidade, normalmente circunscrita por um halo radiolúcido bem definido ${ }^{4,11}$.

Histologicamente, é descrito um padrão de trabéculas ósseas irregulares espessas e com patente pavimentação osteoblástica, e o estroma ricamente celularizado e fasciculado ${ }^{12}$.

O tratamento para esta lesão ainda se mantém controverso na literatura, sendo o prognóstico incerto. Muitos autores sugerem a ressecção em bloco preferível em relação ao tratamento conservador ${ }^{6}$. Alguns autores sugerem a curetagem como o método de tratamento aplicado para lesões bem definidas e pequenas ${ }^{5}$. Lesões maiores e bem definidas devem ser enucleadas. Ressecções devem ser resguardadas para casos extensos, agressivos ou recidivantes. Porém, quanto antes diagnosticada e removida a lesão, melhor é o prognóstico do paciente ${ }^{5,13}$. A frequência de recorrência dessas lesões tratadas com ressecções é extremamente baixa $^{14,15}$

\section{CONCLUSÃO}

Os fibromas ossificantes centrais apresentam na literatura possibilidades variadas de tratamento. Entretanto, não se tem ainda um padrão ouro ou algum protocolo a ser seguido, por tal motivo, os casos devem ser planejados e estudados para serem tratados de forma individualizada e de acordo com a vivência do cirurgião, haja vista que por apresentarem curso benigno as ressecções amplas devem ser ponderadas, evitando assim mutilações dos pacientes, preservando funcionalidade e estética.

\section{REFERÊNCIAS}

01. Speight PM, Takata T. New tumour entities in the 4th edition of the World Health Organization Classification of Head and Neck tumours: odontogenic and maxillofacial bone tumours. Virchows Arch. 2018; 472(3):331-339.

02. Woo SB. Central Cemento-Ossifying Fibroma: Primary Odontogenic or Osseous Neoplasm? J Oral Maxillofac Surg. 2015; 73(12):87-93.

03. Singh AK, Kundendu BA, Sagari SG. Understanding Massive Maxillofacial Central Cement-Ossifying Fibroma. Indian Journal of Stomatology. 2014; 5(4):148-150.

04. MacDonald DS. Maxillofacial fibro-osseous lesions. Clin Radiol. 2015; 70(1):25-36.

05. Dominguete, $\mathrm{MH}$ et al. Extensive presentation of central ossifying fibroma treated with conservative surgical excision. Case Rep Dent. 2014; $1-4$.

06. Tortorici S, Buzzanca ML, Burruano F, Difalco P. Juvenile central ossifying fibroma of the mandible: a case report. J Pediatr Surg. 2010; 45(10):e1-4.

07. Desai K, Gupta K, Manjunatha BS, Palan S. Bimaxillary presentation of central ossifying fibroma: a unique aggressive entity. BMJ Case Rep. 2013; 16:1-4.

08. Misra SR, Saigal A, Rastogi V, Priyadarshini SR, Pati AR. Giant central ossifying fibroma of the maxilla presenting with a pus discharging intra-oral sinus. J Clin Diagn Res. 2015; 9(1): 8-11.

09. Sarwar HG, Jindal MK, Ahmad S. A case report of cemento-ossifying fibroma. J Maxillofac Oral Surg. 2010; 9(2):178-181.

10. Han J. et al. Juvenile ossifying fibroma of the jaw: a retrospective study of 15 cases. Int J Oral Maxillofac Surg. 2016; 45(3):368-376.

11. Mortazavi H, Baharvand M, Rahmani S, Jafari, Parvaei P. Radiolucent rim as a possible diagnostic aid for differentiating jaw lesions. Imaging Sci Dent. 2015; 45(4):253-261. 
12. Moshy JL, Mwakyoma HA, Chindia ML. Evaluation and histological maturation characteristics of fibrous dysplasia and ossifying fibroma: a case series. East Afr Med J. 2010; 87(5):215-219.

13. Titinchi F, Morkel J. Ossifying Fibroma: Analysis of Treatment Methods and Recurrence Patterns. J Oral Maxillofac Surg. 2016; 74(12):2409-2419.
14. Triantafillidou K, Venetis G, Karakinaris G, Iordanidis F. Ossifying fibroma of the jaws: a clinical study of 14 cases and review of the literature. Oral Surg Oral Med Oral Pathol Oral Radiol. 2012; 114(2):193-199.

15. Maria A, Sharma Y, Malik M. Juvenile ossifying fibroma of mandible: a case report. J Maxillofac Oral Surg. 2013; 12(4): 447-450.

\section{ABSTRACT}

Objective: The central ossifying fibroma, according to WHO's latest classification, is an odontogenic tumour, originated in the periodontal ligament cells. Most of the lesions are found in regions near the highest frequency teeth in the mandible. There was female predominance, specially on the early adulthood. Imaging test results and/or facial asymmetries are the chief reasons to have harbour suspicions. The diagnosis should include information obtained from anamnesis, and furthermore imaging and histopathological examinations that confirm the nature of the lesion. The treatment might be held differently, from tissue's lesion curettage to en bloc resection, depending on the size of the damage and its location. The authors describe the case of a 13-yearold female patient with unilateral mandible injury. Materials and methods: The suggested treatment, after the confirmation of the lesion by incisional biopsy, was an exeresis of the lesion preserving the mental nerve, under local anesthesia. After six months of constant reassessments, there was persistence of the lesion, but presenting alterations through the new histopathological test. Results: The absence of lesion injury and the age of the patient, along with her bone development, leaded to the proservation of the case, with semi-annual reexamination. Conclusions: Fibroosseous lesions must be treated severally, in conformity with the surgeon's experience. Considering that it presents benign course, the broad resections might be pondered, being adamant to discuss the adequate conduct with such injuries.

KEYWORDS: Ossifying fibroma; Mandible; Neoplasms.

\author{
AUTOR PARA CORRESPONDÊNCIA \\ Lucas Teixeira Brito \\ Departamento de Cirurgia e Traumatologia \\ Buco-Maxilo-Facial \\ Hospital Estadual Governador Otávio Lage de Siqueira \\ $\left(\mathrm{HUGOL}^{+}\right.$) \\ Av. Anhanguera 14527, CEP: 74463-350, Goiânia, Goiás, \\ Brasil \\ Telefone: +55 62983167163 \\ E-mail: lucasteixeira.95@hotmail.com
}

